\title{
A theoretical approach to the tight-binding band structure of liquid carbon and silicon beyond linear approximations
}

Cite as: J. Chem. Phys. 106, 10238 (1997); https://doi.org/10.1063/1.474050

Submitted: 06 March 1997. Accepted: 21 March 1997. Published Online: 31 August 1998

E. Lomba, J. L. López-Martín, J. A. Anta, J. S. Ho/ye, and G. Kahl

ARTICLES YOU MAY BE INTERESTED IN

Liquid theory for band structure in a liquid

The Journal of Chemical Physics 91, 5613 (1989); https://doi.org/10.1063/1.457564

Ultrafast electron diffraction from non-equilibrium phonons in femtosecond laser heated Au films

Applied Physics Letters 108, 041909 (2016); https://doi.org/10.1063/1.4940981

Ultrafast carrier thermalization and trapping in silicon-germanium alloy probed by extreme ultraviolet transient absorption spectroscopy

Structural Dynamics 4, 044029 (2017); https://doi.org/10.1063/1.4985056

\section{Lock-in Amplifiers up to $600 \mathrm{MHz}$}
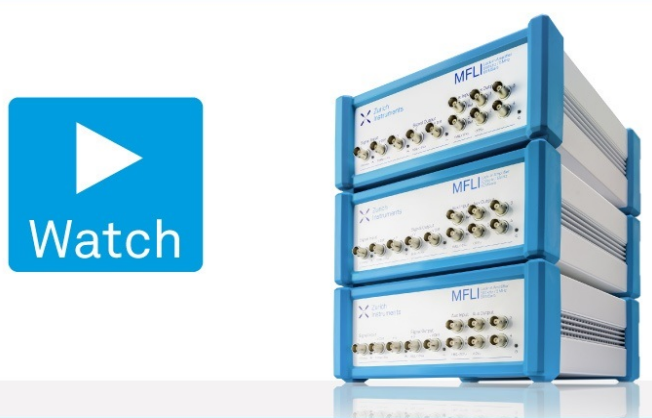

J. Chem. Phys. 106, 10238 (1997); https://doi.org/10.1063/1.474050

106, 10238

(c) 1997 American Institute of Physics. 


\title{
A theoretical approach to the tight-binding band structure of liquid carbon and silicon beyond linear approximations
}

\author{
E. Lomba, J. L. López-Martín, and J. A. Anta \\ Instituto de Química Física Rocasolano, CSIC, Serrano 119, E-28006 Madrid, Spain \\ J. S. Høye \\ Gruppe for Teoretisk Fysikk, Institutt for Fysikk, NTNU, N-7034 Trondheim, Norway \\ G. Kahl \\ Institut für Theoretische Physik, Technische Universität Wien and CMS, Wiedner Hauptstrasse 8-10, \\ A-1040 Wien, Austria
}

(Received 6 March 1997; accepted 21 March 1997)

\begin{abstract}
We present a study of the band structure of liquid Carbon and Silicon modelled in a Tight-Binding Hamiltonian approximation by means of an integral equation approximation that includes non-linear corrections. The theoretical predictions are contrasted with Tight Binding Molecular Dynamics simulations in which the energy bands are obtained by direct diagonalization of the Hamiltonian matrix. The results for Silicon are excellent, whereas in liquid Carbon only some of the qualitative features of the band structure are captured by the non-linear corrections. We find that this can largely be understood as an effect of missing three-body correlation functions in the theoretical treatment of the energy bands. This is particularly crucial in the case of strongly directional and short range bonding, as it occurs in Carbon. (C) 1997 American Institute of Physics. [S0021-9606(97)51124-X]
\end{abstract}

\section{INTRODUCTION}

In a recent paper Lomba, López-Martín and Kahl ${ }^{1}$ investigated the band structure of a rather academic model for liquid $\mathrm{Hg}$ in terms of the Single Superchain/Effective Medium Approximation (SSCA/EMA), a theory that turned out to yield reasonably good results when compared with direct diagonalization of the Tight-Binding (TB) Hamiltonian over quenched liquid configurations. Later, the same authors in collaboration with Winn and Rassinger ${ }^{2}$ presented an application of the same approach to determine the band structure of liquid Silicon. In this case, the electronic structure was modelled by means of an Empirical Tight-Binding Hamiltonian following the parameterization of Goodwin et al. ${ }^{3}$ Also here it was found that the linear SSCA/EMA theory produced results in good agreement with the simulated Tight Binding Molecular Dynamics (TBMD) band structure. Nonetheless, in both cases it became clear that some features of the band structure were not correctly captured by the theory, in particular the high energy wings of the band, which is a well known drawback of linear approximations. ${ }^{4,5}$ It is thus of primary importance to develop non-linear corrections which while retaining the simple structure of the SSCA/EMA for the $s p^{3}$ ( $s$ - and $p$-band) system can cope with the deficiencies inherent to the linear approach. In this connection, Høye and Lomba ${ }^{6-8}$ developed an approximation that accounts for the correct two-particle contribution missing in the low density limit of the Mean Spherical Approximation (MSA) (which is nothing but the SSCA/EMA with the pair distribution function of the quenched fluid replaced by zero outside the hard core diameter ${ }^{9,10}$ ) for a system of coupled Drude oscillators. As pointed out in Ref. 1 the problem of the Tight-Binding bands in an $s p^{3}$ fluid is fully equivalent to a system of coupled three dimensional
Drude oscillators with appropriate coupling functions, and thus the formalism developed in Ref. 8 will apply here as well. The corrections obtained reproduce the presence of high energy wings in the band, which is mainly an effect of particle pairs, as also will be seen in this work. Moreover, they are constructed on top of the MSA, which is linear as the SSCA/EMA, and hence we can keep the core of the computational scheme introduced in Ref. 1 intact in our new approach (the portion that pertains to the Ornstein-Zernike like equation). In the case of the $s p^{3}$ system we will see that certain simplifications derived from the linearity of the closure no longer hold, in particular the transversal and longitudinal contributions to the $p$-band will not be identical any more, and this implies that a certain reformulation of some of the equations has to be done.

As test cases for our non-linear approximation we have chosen liquid silicon, for which it was already seen that the linear approximation works well, ${ }^{2}$ and liquid carbon, a system which was studied some time ago by Tight Binding Molecular Dynamics by Wang et al. ${ }^{11}$ In this case, by simple inspection of the form of the hopping integrals, we know that deviations from mean field behavior will be important. (The range of the interaction is half that of the silicon, and the value of the hopping elements at nearest neighbor distance is doubled.) This can be further confirmed by analyzing the TB density of states (DoS) obtained by Wang et al. ${ }^{11}$ Liquid Carbon will thus be a stringent test for our non-linear corrections, which are the leading ones for interactions of long range.

In summary, we will here present an application of the non-linear corrections proposed by Høye and Lomba ${ }^{6}$ to the multi-band problem posed by disordered systems with $s p^{3}$ TB Hamiltonians. These corrections, that are mostly domi- 
nant for relatively low densities, are exact as concerns pairs of particles. The explicit form of these corrections will be presented in Section II. Notice that in Refs. 6-8 notation and language are somewhat different to that used in Refs. 1 and 2 which follow closely Winn and Logan's Green function formalism. ${ }^{9,10}$ Since the corrections are more easily introduced in the context of the Drude oscillator problem used in Ref. 6 we will follow in Section II this notation and then in Section III we will present a clear mapping between both formalisms. From thereon we will retake the language of the Green's function approach. The reformulation of the equations needed by the symmetry breaking effects of the nonlinearity (or of the use of different on-site energies for the $p p_{\sigma}$ and $p p_{\pi}$ Hamiltonian elements) is also discussed in this section. Finally, in Section IV we present the results of the theoretical approach and compare them with TBMD data. Here we will also explore the use of direct diagonalization of the TB Hamiltonian matrix while averaging over quenched configurations generated by means of classical potentials that incorporate three body interactions, like the Stillinger-Weber (StW) potential for Silicon ${ }^{12}$ and Tersoff's potential for Carbon. ${ }^{13} \mathrm{~A}$ brief discussion about the effect on the electronic structure by the use of empirical three-body interactions instead of the self-consistent TB band energy produced by the TBMD, is also included and will illuminate the role of the spatial ordering on the electronic structure of these systems.

\section{NON-LINEAR CORRECTIONS TO THE FREQUENCY SPECTRUM OF A FLUID OF COUPLED VECTOR DRUDE OSCILLATORS}

As described in Ref. 1 the band structure problem of a fluid whose electronic structure is described by means of an $s p^{3}$ TB Hamiltonian is formally equivalent to the description of the frequency spectrum of a system of particles with embedded three-dimensional Drude oscillators that are coupled. Also, as pointed out in Ref. 1 the symmetry of the electronic interactions will be equivalent to those of an ion-dipole mixture. The corresponding correlation functions at the level of the MSA can be written as

$$
\begin{aligned}
& c_{s s}(12)=c_{s s}^{000}(r) \frac{s_{1_{0}} s_{2_{0}}}{\left\langle s_{0}^{2}\right\rangle}, \\
& c_{s p}(12)=c_{s p}^{011}(r)\left(\hat{r} \hat{s}_{2}\right) \frac{s_{1_{0}} s_{2}}{\sqrt{\left\langle s_{0}^{2}\right\rangle\left\langle s_{2}^{2}\right\rangle}}, \\
& c_{p s}(12)=c_{p s}^{101}(r)\left(\hat{r} \hat{s}_{1}\right) \frac{s_{1} s_{2_{0}}}{\sqrt{\left\langle s_{0}^{2}\right\rangle\left\langle s_{1}^{2}\right\rangle}}, \\
& c_{p p}(12)=c_{p p}^{110}(r) \Phi^{110}(12)+c_{p p}^{112} \Phi^{112}(12) \frac{s_{1} s_{2}}{\left\langle s^{2}\right\rangle},
\end{aligned}
$$

where

$$
\begin{aligned}
& \phi^{110}(12)=\hat{\mathbf{s}}_{1} \hat{\mathbf{s}}_{2} \\
& \phi^{112}(12)=3\left(\hat{\mathbf{r}} \hat{\mathbf{s}}_{1}\right)\left(\hat{\mathbf{r}} \hat{\mathbf{s}}_{2}\right)-\hat{\mathbf{s}}_{1} \hat{\mathbf{s}}_{2}
\end{aligned}
$$

Due to symmetry $c_{p s}^{101}(r)=-c_{s p}^{011}(r)$. The $s_{i_{0}}(i=1,2)$ is the scalar amplitude of the Drude oscillator associated with the $s$-orbital, and $\mathbf{s}_{i}$ are the vector quantities describing the direction and amplitude of the vectorial oscillator associated with the $p$-orbitals. Furthermore $\mathbf{r}$ is the relative distance between the two particles, and the hatted quantities denote unit vectors. Now, according to Eq. (2.14) of Ref. 8 (with $\left.g\left(m^{2}\right) \rightarrow D(E)\right)$, the density of states will be given by

$$
D(E)=-\lim _{\chi \rightarrow 0^{+}} \frac{1}{\pi} \operatorname{Im}\left\{\frac{1}{\rho_{0}} \operatorname{Tr}\left(\boldsymbol{\sigma} \mathbf{R}_{K}\right)\right\},
$$

where $\rho_{0}$ is the total number density of particles, $\boldsymbol{\sigma}$ is a diagonal matrix whose elements are $\sigma_{i i}=M_{i} / \hbar^{2}$, with $M_{i}$ the reduced "mass" of each oscillator and $K^{2}=-\hbar^{2} \omega^{2}+i$. Having a four-band problem we will have four reduced "masses" that will account for the different on-site energies that correspond to each atomic orbital involved. The energy is connected to the frequency through $E=\hbar^{2} \omega^{2}+$ const. in the equivalent harmonic oscillator problem. The crucial quantity in (2.3) is $\mathbf{R}_{K}$, where the $K=-i \hbar \omega$ is the imaginary frequency introduced by the path integral formalism for quantized oscillators. As shown in Ref. $6, \mathbf{R}_{K}$ contributes to the internal energy in a way that turns out to be precisely what the Green's function for the TB Schrödinger equation does in Winn and Logan's formalism.

Now, if we incorporate corrections beyond the MSA in Ref. 8 it was shown that for low density (see Eq. (2.16) therein)

$$
\begin{aligned}
R_{K_{i j}}= & \int \rho_{s} s_{i} s_{j} d \mathbf{s}+\int \rho_{s_{1}} \rho_{s_{2}} s_{1_{i}} s_{1_{j}} \\
& \times\left[\frac{e^{v}}{G(r)}-\frac{1}{2} v^{2}-v-1\right] d \mathbf{s}_{1} d \mathbf{s}_{2} d \mathbf{r},
\end{aligned}
$$

where $\rho_{s}$ is the density of "free" particles and $v=\mathbf{s}_{1_{i}} \mathscr{C}_{i j}(12) \mathbf{s}_{2_{j}}$ with $\mathscr{C}_{i j}$ denoting the chain bond as defined in Refs. 6 and 8. Here and in the rest of this part the vector $\mathbf{s}$ also includes the fourth component $s_{0}$ of Eq. (2.1), i.e., $i, j=0$ is included. To leading order $v$ is the MSA pair correlation function. The configurational function $G(r)$ is a quenching factor that was introduced to cancel out the effect of induced correlations that otherwise would originate from the internal degrees of freedom when using statistical mechanical theory of systems at thermal equilibrium. Here we note that according to the ideas of Ref. 6 the leading nonlinear contribution to the frequency spectrum will stem from the formation of "diatomic" particle pairs. The chemical equilibrium between these "diatomic" species and the atomic "free" species will be governed by a law of mass action (Eq. (2.6) in Ref. 6). It must be recalled here that in accordance with this picture first introduced by Onsager, a continuous degree of freedom will be equivalent to a mixture of different species. The first term in Eq. (2.4) gives the 
MSA type linear contribution to the frequency spectrum, while the second gives the one from particle pairs. Now, if one incorporates the law of mass action and the quenching factor $G(r)$ in Eq. (2.4) (Eq. (2.16) in Ref. 6) one can also write

$R_{K_{i j}}=\frac{\rho}{\rho_{f}} \int \rho_{s} s_{i} s_{j} d s+\rho^{2}\left(\mathbf{a}^{-1} \mathbf{D}_{1}\right)_{i j}-\rho^{2}\left(\mathbf{a}^{-1} \mathbf{D}_{2}\right)_{i j}$,

where we have defined

$$
\rho_{f} \mathbf{a}_{i j}^{-1}=\int \rho_{s} s_{i} s_{j} d s,
$$

by which one gets

$$
\mathbf{R}_{K}=\rho \mathbf{a}^{-1}\left[\mathbf{I}+\rho \mathbf{D}_{1}-\rho \mathbf{D}_{2}\right] .
$$

Here $\rho$ is the total density ( $\rho_{0}$ of Refs. 6 and 8) while $\rho_{f}$ is the density of free particles ( $\rho$ of Refs. 6 and 8). The prefactor $\rho / \rho_{f}$ in Eq. (2.5) compensates the $\left\langle s_{1 i} s_{1 j}\right\rangle$ term included in $D_{2}$ as explained below (2.17). The $\mathbf{R}_{K}$ can be approximated or replaced by

$$
\mathbf{R}_{K}=\rho\left[\mathbf{a}_{e}^{-1}+\rho \mathbf{a}^{-1} \mathbf{D}_{1}\right],
$$

where the effective $\mathbf{a}_{e}=\mathbf{a}+\rho \mathbf{D}_{2} \mathbf{a}$. Due to the symmetry of the $s p$ and $p s$ interactions, the off-diagonal elements of $\mathbf{R}_{K}$ must satisfy $\mathbf{R}_{K}^{s p}=-\mathbf{R}_{K}^{p s}$, and this equality is broken if one rewrites Eq. (2.8) precisely as was done in Eq. (4.6) of Ref. 6 . If the symmetry condition is broken at some stage of the iterative process of solution, the numerical stability will be badly affected, therefore we will retain here the form of Eq. (2.8) in our calculation in contrast to the scheme followed in Refs. 7 and 8. The first correction term in Eqs. (2.5) or (2.7) will be

$$
\rho^{2}\left[\mathbf{a}^{-1} \mathbf{D}_{1}\right]_{i j}=\int \rho_{s_{1}} \rho_{s_{2}} \hat{s}_{1_{i}} \hat{s}_{1_{j}}\left[\frac{e^{v}}{G(r)}-v-1\right] d \hat{s}_{1} d \hat{s}_{2} d \mathbf{r} .
$$

The Gaussian type integration (2.9) can be performed, and one gets

$$
\rho^{2}\left[\mathbf{a}^{-1} \mathbf{D}_{1}\right]_{i j}=\int \rho^{2}\left[\mathbf{M}^{-1}-\mathbf{M}_{0}^{-1}\right]_{i j}^{11} d \mathbf{r},
$$

where the two particle $\mathbf{M}$ matrix is composed of $4 \times 4$ submatrices, such that

$$
\mathbf{M}=\left(\begin{array}{ll}
\mathbf{M}^{11} & \mathbf{M}^{12} \\
\mathbf{M}^{21} & \mathbf{M}^{22}
\end{array}\right) \text {. }
$$

The submatrices involved are

$$
\mathbf{M}^{11}=\mathbf{M}^{22}=\mathbf{a}
$$

and

$$
\mathbf{M}^{12}=\mathbf{M}^{21}=\left(\begin{array}{cc}
\mathscr{C} & 0 \\
0 & \mathscr{C}^{\prime}
\end{array}\right) .
$$

The chain bond matrices can be decomposed in their elements according to the spherical harmonic projections in a reference frame with the $z$-axis joining the particle centers, also called axial frame ${ }^{1}$ (i.e. lower indices vs. upper indices).
The relation between the axial and spatial coefficients (those defined in a space fixed reference frame) is given by

$$
\begin{aligned}
& \mathscr{C}_{000}^{s s}=\mathscr{C}_{s s}^{000}, \quad \mathscr{C}_{010}^{p p}=\mathscr{C}_{s p}^{011} / \sqrt{3}, \\
& \mathscr{C}_{110}^{p p}=\frac{1}{3}\left[\mathscr{C}_{p p}^{110}+2 \mathscr{C}_{p p}^{112}\right], \quad \mathscr{C}_{111}^{p p}=\frac{1}{3}\left[\mathscr{C}_{p p}^{112}-\mathscr{C}_{p p}^{110}\right] .
\end{aligned}
$$

This expressions will hold for the expansion coefficients of all correlation functions involved in this treatment. With this one gets for the chain bond

$$
\mathscr{C}=\left(\begin{array}{cc}
\mathscr{C}_{000}^{s s} & \mathscr{C}_{010}^{p p} \\
\mathscr{C}_{100}^{p s} & \mathscr{C}_{110}^{p p}
\end{array}\right) \text { and } \mathscr{C}^{\prime}=\left(\begin{array}{cc}
\mathscr{C}_{111}^{p p} & 0 \\
0 & \mathscr{C}_{111}^{p p}
\end{array}\right)
$$

As mentioned above, the $\mathscr{C}_{\mathrm{klm}}^{\alpha \beta}$ are the coefficients of the spherical harmonic expansion of the chain bond functions in a reference frame in which the $z$-axis lies along $\mathbf{r}$. The quantity $\mathbf{M}_{0}$ appearing in Eq. (2.10) is nothing but $\mathbf{M}$ with the chain bonds switched off, and hence

$$
\left[\mathbf{M}_{0}\right]^{11}=\left[\mathbf{M}_{0}\right]^{22}=\mathbf{a}, \quad \text { and }\left[\mathbf{M}_{0}\right]^{12}=\left[\mathbf{M}_{0}\right]^{21}=0 .
$$

Thus, by straightforward inversion of the block diagonal matrix (2.11) and with use of the expression (2.10), one gets the first correction term in $\mathbf{R}_{K}$. As was shown in Ref. 7, the $\mathbf{D}_{1}$ term will be responsible for the appearance of the wings of the band, that are absent in the linear approximations. Now, to compute the second contribution, we have from Ref. 8 that

$$
\rho^{2}\left[\mathbf{a}^{-1} \mathbf{D}_{2}\right]_{i j}=\int \rho_{s_{1}} \rho_{s_{2}}\left(\mathbf{s}_{1_{i}} \mathbf{s}_{1_{j}}-\left\langle\mathbf{s}_{1_{i}} \mathbf{s}_{1_{j}}\right\rangle\right) \frac{1}{2} v^{2} d \mathbf{s}_{1_{i}} d \mathbf{s}_{2_{j}} d \mathbf{r} .
$$

The term between angular brackets in the integrand includes the effect of the equilibrium constant of the pairing reaction, $K_{\mathrm{s}_{1} \mathbf{s}_{2}}$. This can be concluded from Eqs. (2.16) and (2.20) of Ref. 6 and Eq. (2.16) of Ref. 8. From the former two equations it follows that only the $\frac{1}{2} v^{2}$ term in $K_{\mathbf{s}_{1} \mathbf{s}_{\mathbf{2}}}$ gives a net contribution to the total density. The first term of the latter equation represents only the density of "free", particles while the first term of Eq. (2.7) means total density. So the second term in Eq. (2.17) compensates for this difference. After some algebra one gets

$$
\rho^{2} \mathbf{a}^{-1} \mathbf{D}_{2}=\rho^{2} \int \mathbf{a}^{-1} \mathbf{M}^{12} \mathbf{a}^{-1} \mathbf{M}^{12} \mathbf{a}^{-1} d \mathbf{r}
$$

which, as defined below Eq. (2.8) gives us the effective quantity

$$
\mathbf{a}_{e}=\mathbf{a}+\rho \int \mathbf{M}^{12} \mathbf{a}^{-1} \mathbf{M}^{12} d \mathbf{r} .
$$

Note that even if $\mathbf{a}$ is still a diagonal quantity, this no longer holds for its effective counterpart, since the chain bond matrix incorporates the $s p$ correlations. Now, by insertion of Eqs. (2.10) and (2.19) into Eq. (2.8) and using Eq. (2.3) we get an energy spectrum that incorporates non-linear corrections, provided we know how to calculate the chain bond elements. In principle one can calculate them in an iterative procedure following the scheme of the Exponential Hyper- 
vertex Approximation, ${ }^{14}$ but this can be an ill-conditioned problem. Therefore, following the lines of Refs. 7 and 8 we will here simply approximate the chain bond functions by their values in the MSA, which are nothing but the MSA total correlation functions, $h^{\alpha \beta}(12)$. This will only be done in the non-linear terms. Otherwise we use the OrnsteinZernike (OZ) equation with the SSCA linear closure. Some modifications with respect to the equations in Ref. 1 will have to be implemented at this level, to account for the splitting of the transversal and longitudinal components of $\mathbf{R}_{K}$, in particular concerning the coefficients of the elements of $\mathbf{R}_{K}$ entering the $\mathrm{OZ}$ equation.

\section{NON-LINEAR CORRECTIONS IN WINN-LOGAN'S FORMALISM}

Now, if we want to incorporate the corrections introduced in the previous section into the formalism used in Refs. 1 and 2 we have to carefully map the needed quantities. When comparing Eq. (2.3) for the energy spectrum with the corresponding expression for the density of states evaluated from the average diagonal Green's function,

$$
D(E)=\lim _{\xi \rightarrow 0^{+}} \frac{1}{\pi} \operatorname{Im}\{\operatorname{Tr} \mathbf{G}(E+i \xi)\}
$$

one sees that

$$
\mathbf{R}_{K} / \rho=\mathbf{G}(E),
$$

where we know that $\sigma K^{2} \leftrightarrow E+$ const.; $\sigma=M / \hbar^{2}$. Then, our energy equation (2.8) with non-linear corrections (Eqs. (3.3) and (3.4) in Ref. 1) will read

$$
\mathbf{G}(z)=\mathbf{a}_{e}^{-1}+\rho \mathbf{a}^{-1} \mathbf{D}_{1},
$$

where

$$
\mathbf{a}=\left(\begin{array}{cccc}
a_{s s} & \ldots & \ldots & 0 \\
\vdots & a_{p p_{\sigma}} & & \vdots \\
\vdots & & a_{p p_{\pi}} & \vdots \\
0 & \ldots & \ldots & a_{p p_{\pi}}
\end{array}\right),
$$

with elements,

$$
\begin{aligned}
& a_{s s}(z)=a_{0_{s s}}(z)+c_{000}^{s s}(0)=\left(\varepsilon_{s}-z\right)+c_{000}^{s s}(0), \\
& a_{p p_{\sigma}}(z)=a_{0_{p p_{\sigma}}}(z)+c_{110}^{p p_{\sigma}}(0)=\left(\varepsilon_{p_{\sigma}}-z\right)+c_{110}^{p p}(0), \\
& a_{p p_{\pi}}(z)=a_{0_{p p_{\pi}}}(z)-c_{111}^{p p_{\pi}}(0)=\left(\varepsilon_{p_{\pi}}-z\right)-c_{111}^{p p}(0),
\end{aligned}
$$

where $z=E+i \xi$, and we have assumed that the on-site Hamiltonian elements $\varepsilon_{p_{\sigma}}$ and $\varepsilon_{p_{\pi}}$ might be different depending on the type of TB parameterization. ${ }^{15}$ In Eq. (3.5) the $c_{k l m}^{\alpha \beta}$ are the spherical harmonic expansion coefficients of the direct correlations functions corresponding to the internal degrees of freedom, which are connected with those of Eqs.
(2.1) by transformations (2.14). Now, since a general property of these expansion coefficients is that $c_{111}(0)=-c_{110}(0)$, it is clear that when $\varepsilon_{p_{\sigma}}=\varepsilon_{p_{\pi}}$ then $a_{p p_{\sigma}}=a_{p p_{\pi}}$, thus establishing the equality between the longitudinal and transversal components of $\mathbf{G}^{p p}$ when the closure is linear. In any case, for the systems to be dealt with here, the three $p$-components of a (but not of $\mathbf{G}$ ) will be identical, although we will keep the different notation for the sake of generality. Now, we still have to find the values of $c_{k l m}^{\alpha \beta}(0)$ but this is a straightforward task if one recalls that the $h_{k l m}^{\alpha \beta}(r)=0$ for $r$ inside the overlap region, and uses the $\mathrm{OZ}$ relation (Eq. (3.16) in Ref. 1). Note that here we use lower case $h$ and $c$ for the total and direct correlation functions, to avoid confusion with the chain bond function $\mathscr{C}$. The $c_{k l m}^{\alpha \beta}(0)$ can now be expressed in terms of convolutions of $\mathbf{h}$ and $\mathbf{c}$. In the MSA case one will simply have $\mathbf{a}_{e}=\mathbf{a}$,

$$
\mathbf{G}^{\mathrm{MSA}}=\mathbf{a}^{-1},
$$

and the $c_{k l m}^{\alpha \beta}(0)$ in Eq. (3.5) expressed as convolutions via the OZ equation is precisely the energy as in Eq. (3.22) of Ref. 1. (Note that in the MSA $c_{k l m}^{\alpha \beta}(r)=V_{k l m}^{\alpha \beta}(r)$ outside the particle core, where $V$ represents the interaction.) But if we want to go beyond the linear approximation we just have to retain the chain bond terms in $\mathbf{a}_{e}$ and calculate $\mathbf{D}_{2}$, which as already mentioned will no longer be diagonal. When going beyond the low density limit, we can introduce finite density corrections along the line suggested in Ref. 8, which modifies Eq. (2.19) into

$$
\mathbf{a}_{e}=\mathbf{a}+\frac{1}{\rho} \int \mathbf{M}^{12} \mathbf{a}^{-1} \mathbf{M}^{12}\left[\left(\mu^{2}-\rho^{2}\right) g(r)+\rho^{2}\right] d \mathbf{r},
$$

where $g(r)$ is the pair distribution function of the quenched fluid and $\mu=\rho(\partial \beta P / \partial \rho)^{-1}$, i.e. $\mu / \rho$ is the bulk isothermal compressibility. The $\mathbf{D}_{1}$ term requires some further elaboration. With arbitrary density corrections we can rewrite Eq. (2.10) as

$$
\rho \mathbf{a}^{-1} \mathbf{D}_{1}=4 \pi \frac{\mu^{2}}{\rho} \int \boldsymbol{\Delta}(r) g(r) r^{2} d r
$$

where we have defined the quantity

$$
\boldsymbol{\Delta}(r)=\left[\mathbf{M}^{-1}\right]^{11}-\left[\mathbf{M}_{0}^{-1}\right]^{11},
$$

which by inversion of Eq. (2.11) can be explicitly written as

$$
\Delta(r)=\left(\begin{array}{ccc}
\boldsymbol{\Delta}_{2 \times 2} & \ldots & 0 \\
\vdots & \Delta_{33} & \vdots \\
0 & \ldots & \Delta_{44}
\end{array}\right),
$$

where by use of computer algebra we find

$$
\Delta_{33}=\Delta_{44}=\frac{a_{p p_{\pi}}}{a_{p p_{\pi}}^{2}-h_{111}^{p p^{2}}}-\frac{1}{a_{p p_{\pi}}}
$$

and 


$$
\Delta_{2 \times 2}=\frac{1}{\delta}\left(\begin{array}{cc}
a_{p p_{\sigma}}^{2} a_{s s}-a_{s s} h_{110}^{p p^{2}}-a_{p p_{\sigma}} h_{100}^{p s} h_{010}^{s p}-\delta / a_{s s} & a_{s s} h_{110}^{p p} h_{010}^{s p}+a_{p p_{\sigma}} h_{000}^{s s} h_{010}^{s p} \\
-\left(a_{s s} h_{110}^{p p} h_{010}^{s p}+a_{p p_{\sigma}} h_{000}^{s s} h_{010}^{s p}\right) & a_{p p_{\sigma}} a_{s s}^{2}-a_{p p_{\sigma}} h_{000}^{s s^{2}}-a_{s s} h_{100}^{p s} h_{010}^{s p}-\delta / a_{p p_{\sigma}}
\end{array}\right)
$$

with

$$
\begin{aligned}
\delta= & a_{p p_{\sigma}}^{2} a_{s s}^{2}-a_{s s}^{2} h_{110}^{p p^{2}}-2 a_{p p_{\sigma}} a_{s s} h_{010}^{s p} h_{100}^{p s}+h_{010}^{s p^{2}} h_{100}^{p s^{2}} \\
& -2 h_{110}^{p p} h_{100}^{p s} h_{010}^{s p} h_{000}^{s s}-a_{p p_{\sigma}}^{2} h_{000}^{s s^{2}}+h_{110}^{p p^{2}} h_{000}^{s s^{2}} .
\end{aligned}
$$

In these expressions we will here use MSA chain bond functions, $h_{k l m}^{\alpha \beta}$. Note that in Eq. (3.10) one can see the familiar form of the denominator of the $D_{1}$ corrections terms, whose poles will give rise to the wings of the spectrum, and from Eqs. (3.9) and (3.11) it follows that the transversal components will be completely decoupled from the longitudinal term, and that the off-diagonal elements of the $\mathbf{G}$ matrix will fulfill $\mathbf{G}^{s p}=-\mathbf{G}^{p s}$. We retain the boldface in the matrix elements of $\mathbf{G}$, since in accordance to the discussion in Ref. 1 these terms will be vectors of the form $\mathbf{G}^{s p}=G^{s p}(1,0,0)$ and similarly for $\mathbf{G}^{p s}$ which now will be a column vector. Let us also recall that the $\mathbf{G}^{p p}$ element will be a $3 \times 3$ diagonal matrix with elements $\left(\mathbf{G}^{p p}\right)_{11}=G_{\sigma}^{p p}$, $\left(\mathbf{G}^{p p}\right)_{22}=\left(\mathbf{G}^{p p}\right)_{33}=G_{\pi}^{p p}$.

In the above paragraph we have run into a rather subtle complication when we now want to translate the r-dependent form of the matrix OZ equations (Eq. (3.7) in Ref. 1) into molecular OZ equations in which the usual rotational invariant expressions can be applied, as was done in Ref. 1. It turns out that, neither $\mathbf{G}^{s p}\left(\mathbf{G}^{p s}\right)$ nor $\mathbf{G}^{p p}$ are the simple rotational invariants considered so far any more, since due to the non-linear terms the $\mathbf{G}^{p p} \neq$ const.I, by which it is not invariant during the rotation operation, which also is the case for $\mathbf{G}^{s p}$. Consequently, when we want to go from the matrix to the angular dependent form of the $\mathrm{OZ}$ equation (i.e. when going from Eq. (3.7) to Eq. (3.10) in Ref. 1) we will have to construct angular functions from the matrix quantities $\mathbf{G}^{s p},\left(\mathbf{G}^{p s}\right)$ and $\mathbf{G}^{p p}$ and perform carefully the convolutions, as was done for the correlation functions. Thus, we will also have functions of the form

$$
\begin{aligned}
& G^{s p}\left(\omega_{3}\right)=\mathbf{G}^{s p} \hat{\mathbf{s}}_{3}, \\
& G^{p p}\left(\omega_{3}\right)=\hat{\mathbf{s}}_{3} \mathbf{G}^{p p} \hat{\mathbf{s}}_{3} .
\end{aligned}
$$

Now, it turns out that a number of new convolution relations will make feasible the connection between the matrix formulation of the OZ equation and the molecular version. As in Ref. 1, we will have for any two matrices (or vectors) $\mathbf{A}$ and B,

$$
\mathbf{A B}=3 \int(\hat{\mathbf{A}} \hat{\mathbf{s}})(\hat{\mathbf{s}} \mathbf{B}) d \omega,
$$

where $\hat{\mathbf{s}}$ is a unit vector whose orientation is described by $\omega$. Also, for any two matrices (or vectors) $\mathbf{A}$ and $\mathbf{B}$ of the appropriate dimensions, and any diagonal matrix $\mathbf{G}^{p p}$

$$
\mathbf{A G}^{p p} \mathbf{B}=\frac{15}{2} \int(\mathbf{A} \hat{\mathbf{s}})\left(\hat{\mathbf{s}} \mathbf{G}^{p p} \hat{\mathbf{s}}\right)(\hat{\mathbf{s}} \mathbf{B}) d \omega-\frac{1}{2} \operatorname{Tr}\left\{\mathbf{G}^{p p}\right\} \mathbf{A B} .
$$

Besides it is easy to write down the explicit angular dependence of $G^{p p}(\omega)$, which is simply given by

$$
G^{p p}(\omega)=\left(G_{\sigma}^{p p}-G_{\pi}^{p p}\right) \cos ^{2} \theta+G_{\pi}^{p p},
$$

where $\cos \theta=\hat{s}_{z}$ and $\mathbf{s}$ is the equivalent harmonic oscillator amplitude for the $p$-band. Now, if one takes into account the aforementioned convolutions and rewrites Eq. (3.7) of Ref. 1 in terms of angular functions which are then expanded in spherical harmonics, only one class of terms will deserve special attention, namely those products of the form

$$
F(12)=\mathbf{s}_{1} \mathbf{A G}^{p p} \mathbf{B s}_{2}
$$

which when expanded in spherical harmonics lead to

$$
\begin{aligned}
F_{k l m}= & (-1)^{m}\left(G_{\sigma}^{p p}+2 G_{\pi}^{p p}\right) \sum_{\lambda} A_{k \lambda m} B_{\lambda l m}+5\left(G_{\sigma}^{p p}-G_{\pi}^{p p}\right) \\
& \times \sum_{\lambda, \chi} \sqrt{(2 \lambda+1)(2 \chi+1)}\left(\begin{array}{ccc}
\lambda & \chi & 2 \\
0 & 0 & 0
\end{array}\right) \\
& \times\left(\begin{array}{ccc}
\lambda & \chi & 2 \\
m & -m & 0
\end{array}\right) A_{k \lambda m} B_{\chi l m}
\end{aligned}
$$

where we have used the convolution relations (3.14) and (3.15), together with Eq. (3.16), and we have performed the substitution

$$
\cos ^{2} \theta=\left(\sqrt{\frac{16 \pi}{5}} Y_{20}(\theta)+1\right) / 3 .
$$

In Eq. (3.18) the quantities

$$
\left(\begin{array}{ccc}
k & l & n \\
m & -m & 0
\end{array}\right)
$$

are 3-J Wigner symbols that occur due to the composition rules of the spherical harmonics. If we now focus on the case $k, l \leqslant 1$, which is the relevant situation for us

$F_{k l 0}=\left(G_{\sigma}^{p p}+2 G_{\pi}^{p p}\right) \sum_{\lambda} A_{k \lambda 0} B_{\lambda l 0}+2\left(G_{\sigma}^{p p}-G_{\pi}^{p p}\right) A_{k 11} B_{1 l 1}$,

$F_{k l 1}=-\left(G_{\sigma}^{p p}+2 G_{\pi}^{p p}\right) \sum_{\lambda} A_{k \lambda 1} B_{\lambda l 1}+\left(G_{\sigma}^{p p}-G_{\pi}^{p p}\right) A_{k 11} B_{1 l 1}$.

With all these relation ${ }^{5}$ it is then possible to get the new $\mathrm{OZ}$ equation, that again, as in Ref. 1 will be decoupled into transversal $(m=1)$ and longitudinal components $(m=0)$ 


$$
\begin{aligned}
& m=0
\end{aligned}
$$

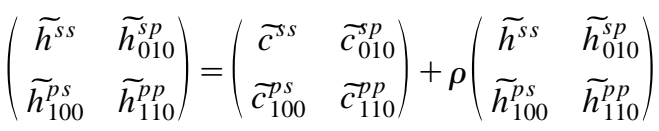

$$
\begin{aligned}
& \times\left(\begin{array}{cc}
G^{s s} & \sqrt{3} G^{s p} \\
\sqrt{3} G^{p s} & 3 G_{\sigma}^{p p}
\end{array}\right)\left(\begin{array}{cc}
\widetilde{c}^{s s} & \widetilde{c}_{010}^{s p} \\
\widetilde{c}_{100}^{p s} & \widetilde{c}_{110}^{p p}
\end{array}\right) .
\end{aligned}
$$

$m=1$

$$
\widetilde{h_{111}^{p p}}=\widetilde{h_{111}^{p p}}-3 \rho G_{\pi}^{p p}{\widetilde{h_{111}^{p p}}}_{c_{111}^{p p}}
$$

The tilde in the above expressions denotes Fourier transform. Now, the SSCA/EMA closure will be

$$
c_{k l m}^{\alpha \beta}=g(r) V_{k l m}^{\alpha \beta}+(g(r)-1)\left[h_{k l m}^{\alpha \beta}-c_{k l m}^{\alpha \beta}\right],
$$

whereas the MSA closure is obtained by replacing the pair distribution function, $g(r)$, by 1 outside a distance that indicates the onset of the overlap region while it is 0 inside. Note that Eq. (3.7) was derived as a leading contribution beyond the MSA. By use of the SSCA/EMA closure (3.23) part of this, namely the $\rho^{2}(g(r)-1)$ term, is already included to a leading order and should thus be deleted to avoid double counting.

Now the coefficients of the electronic interaction are given by

$$
\begin{aligned}
& V_{000}^{s s}=V^{s s}, \quad V_{010}^{s p}=V_{\sigma}^{s p} / \sqrt{3}, \\
& V_{110}^{p p}=V_{\sigma}^{p p} / 3, \quad V_{111}^{p p}=V_{11-1}^{p p}=-V_{\pi}^{p p} / 3 .
\end{aligned}
$$

Explicit expressions for silicon and carbon will be given in the next section. Now, if one solves Eqs. (3.21)-(3.22) with the MSA closure [Eq. (3.23) with a step function for $g(r)$ ] one gets a set correlation functions $h_{k l m}^{\alpha \beta}$ that inserted into Eq. (3.5) will generate the first linear approximation to $\mathbf{G}$. If one goes beyond the linear approximation, one simply has to insert the correlation functions (recall that $\mathscr{C}_{\mathrm{klm}}^{\alpha \beta}$ is approximated by $h_{k l m}^{\alpha \beta}$ ) into Eqs. (3.9), (3.8) and (3.7), to generate the corrections that go into Eq. (3.3) and finally get our nonlinear approximation to $\mathbf{G}$, which is then inserted back into the $\mathrm{OZ}$ equations, and the procedure is repeated until satisfactory convergence is achieved.

\section{AN APPLICATION TO LIQUID SILICON AND CARBON}

As mentioned in the Introduction we have here used the standard Tight Binding Hamiltonian with the empirical parameterization of Goodwin et al. ${ }^{3}$ for Silicon and the model of Wang et al. ${ }^{11}$ for Carbon. The thermodynamic state under consideration for Si corresponds to a temperature of $1740 \mathrm{~K}$ and a density of $2590 \mathrm{~kg} \mathrm{~m}^{-3}$, close to the triple point. For liquid carbon we have considered a thermodynamic state also in the vicinity of the melting transition, namely a temperature of $5000 \mathrm{~K}$ and a density of $2000 \mathrm{~kg} \mathrm{~m}^{-3}$. Explicitly we have dealt with the following parameterization of the TB Hamiltonian,

$$
\begin{array}{ll}
V^{s s}(r)=V^{s s}\left(r_{0}\right) f(r), & V_{\sigma}^{p p}(r)=V_{\sigma}^{p p}\left(r_{0}\right) f(r), \\
V_{\pi}^{p p}(r)=V_{\pi}^{p p}\left(r_{0}\right) f(r), & V_{\sigma}^{s p}(r)=V_{\sigma}^{s p}\left(r_{0}\right) f(r) \\
V_{\sigma}^{p s}(r)=-V_{\sigma}^{s p}(r), &
\end{array}
$$

with

$$
f(r)=\left(\frac{r_{0}}{r}\right)^{2} \exp \left\{2\left[-\left(\frac{r}{r_{c}}\right)^{n_{c}}+\left(\frac{r_{0}}{r_{c}}\right)^{n_{c}}\right]\right\} .
$$

The numerical parameters for silicon were taken from Virkkunen et al., ${ }^{16}$

$$
\begin{aligned}
& V^{s s}\left(r_{0}\right)=-1.820 \mathrm{eV}, \quad V_{\sigma}^{s p}\left(r_{0}\right)=1.960 \mathrm{eV}, \\
& V_{\sigma}^{p p}\left(r_{0}\right)=3.060 \mathrm{eV}, \quad V_{\pi}^{p p}\left(r_{0}\right)=-0.870 \mathrm{eV}, \\
& \varepsilon_{s}=-6.173 \mathrm{eV}, \quad \varepsilon_{p}=2.122 \mathrm{eV}, \\
& r_{c}=3.6 \AA, \quad n_{c}=6.48,
\end{aligned}
$$

$r_{0}=2.35 \AA$ is the equilibrium nearest-neighbor distance of the diamond lattice for $\mathrm{Si}$. In the case of Carbon, we have $\mathrm{e}^{11,17}$

$$
\begin{aligned}
& V^{s s}\left(r_{0}\right)=-5.00 \mathrm{eV}, \quad V_{\sigma}^{s p}\left(r_{0}\right)=4.70 \mathrm{eV}, \\
& V_{\sigma}^{p p}\left(r_{0}\right)=5.50 \mathrm{eV}, \quad V_{\pi}^{p p}\left(r_{0}\right)=-1.55 \mathrm{eV}, \\
& \varepsilon_{s}=-2.99 \mathrm{eV}, \quad \varepsilon_{p}=3.71 \mathrm{eV}, \\
& r_{c}=2.18 \AA, \quad n_{c}=6.5,
\end{aligned}
$$

and $r_{0}=1.536 \AA$ is again the equilibrium nearest-neighbor distance of the diamond lattice. The repulsive energy needed for the TB Molecular Dynamics (but irrelevant to our integral equation approach) is constructed in both cases from a sum of two-center potentials whose explicit expressions can be found in Ref. 16 for silicon and Ref. 17 for carbon.

We have run TB Molecular Dynamics for both systems for 64-particle samples in order to generate reference results and pair correlation functions that served as structural input for the theory. In order to check the sensitivity of the band structure to changes in the structural input we have also determined the structure of liquid Silicon and Carbon by means of classical Monte Carlo simulations in which triplet potentials give account of the particular coordinations dominant in these systems. As already mentioned, we have used the Stillinger-Weber potential ${ }^{12}$ to model liquid silicon and Tersoff's interaction for liquid carbon ${ }^{13}$ (in both cases using 216 particles). In Figs. 1 and 2 it can be seen that whereas the StW potential reproduces reasonably well the TBMD results for the pair structure, there is a considerable discrepancy between the results for Si carbon obtained using Tersoff's potential and TBMD. Apparently, Tersoff's potential leads to nearest neighbor distances too large in liquid phases, and hence one sees a displacement of the position of all the peaks in $g(r)$. Now, if one compares the DoS obtained by diagonalization and averaging for both systems (Figs. 3 and 4), it is clear that the results obtained when using configurations generated according to the StW potential are in reasonably good agreement with TBMD, whereas for liquid carbon the situation is the opposite. On one hand, the displacement of the peaks of $g(r)$ decreases the overlap in the hopping terms, 


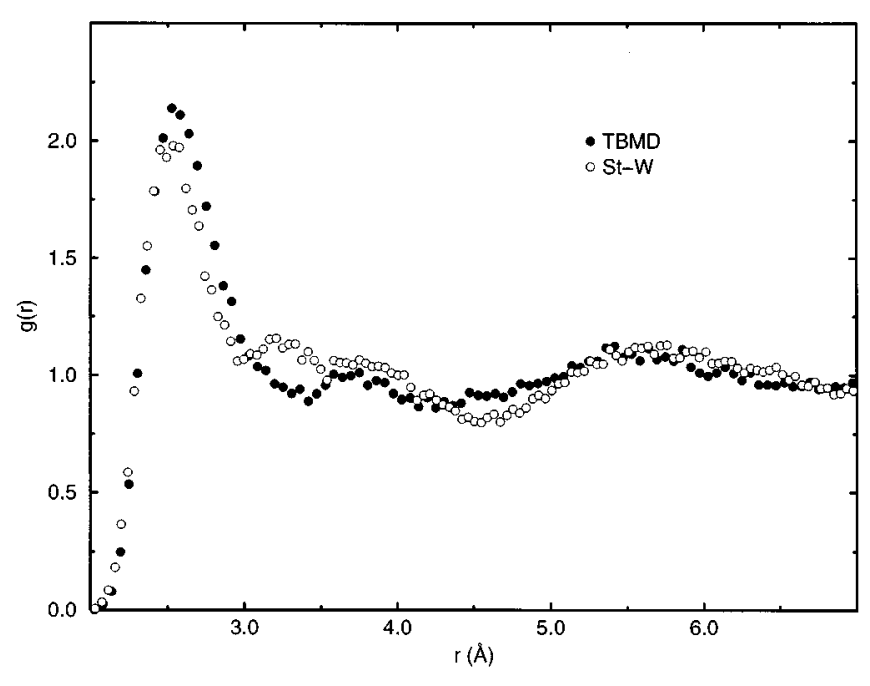

FIG. 1. Pair distribution function for liquid Silicon, obtained from TBMD (filled circles) and Monte Carlo simulation using StW potential (hollow circles).

by which the width of the band shrinks. On the other hand, the central peak ( $p$-band) splits in two in the TBMD results whereas when configurations from Tersoff's potential are used the splitting is missing. A plausible explanation of this difference can be inferred from the value of the average coordination number, which for the TBMD results ${ }^{11}$ is 2.4 and for the Tersoff's potential simulation is in our case 3.4. Somehow, this is telling us that the particles in our TBMD simulations tend to be organized in chains, whereas triplet configurations seem to be more dominant when one uses Tersoff's potential. These features have already been well characterized in the explicit analysis of configurations performed in Refs. 11 and 13 for various states of liquid and amorphous carbon. Thus, in view of our simulation results it seems that the formation of linear chains enhances the split-

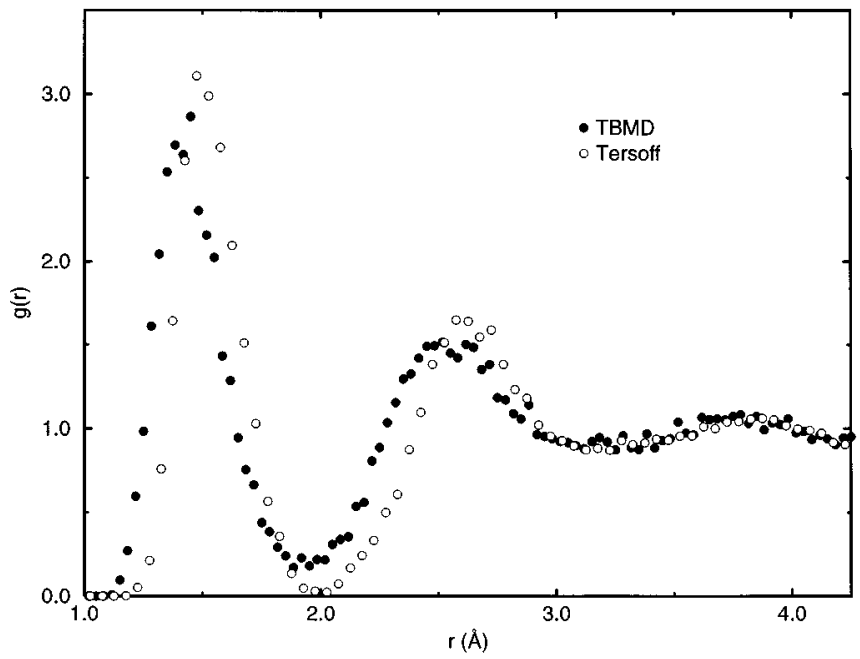

FIG. 2. Pair distribution function for liquid Carbon, obtained from TBMD (filled circles) and Monte Carlo simulation using Tersoff's potential (hollow circles).

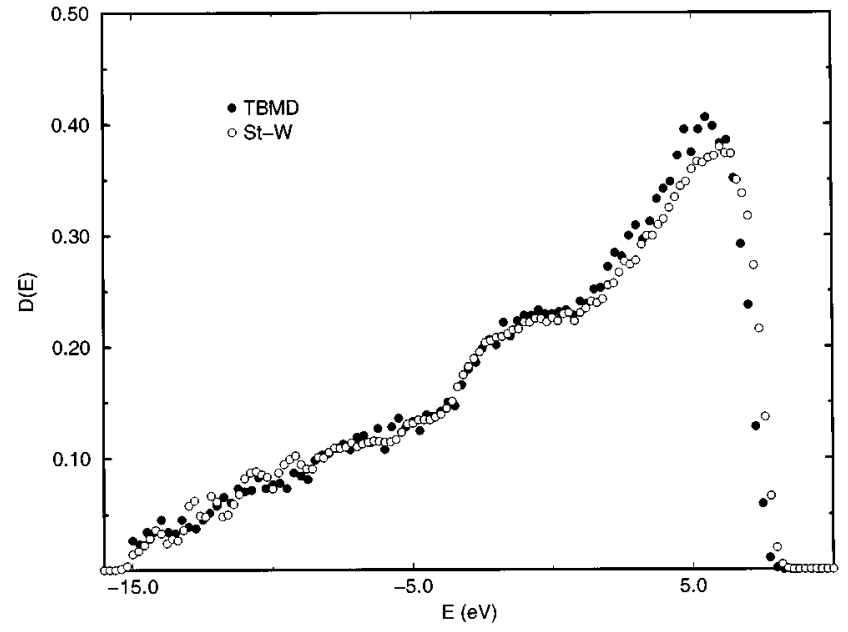

FIG. 3. Total Density of States for liquid Silicon from TBMD (filled circles) and by direct diagonalization over configurations generated by Monte Carlo simulation using StW potential (hollow circles).

ting between $p p_{\sigma}$ and $p p_{\pi}$ levels, as this is what one sees in the $p$-band calculated from the TBMD results.

Now, once we have available structural information, we can proceed to solve the SSCA/EMA and the non-linear approximation. The results for Silicon are presented in Fig. 5. As was found in Ref. ${ }^{2}$ the SSCA/EMA already gives remarkably good results. However, the incorporation of the non-linear terms adds a fine correction to the peak. It has to be pointed out however, that the non-linear terms had to be switched off in the region where the $s$ contribution is dominant to avoid divergence, both when using MSA chain bonds or when these were approximated by the SSCA/EMA $h^{\alpha \beta}$. This type of failure is also present in linear approximations at high density. ${ }^{19}$ In principle, for the value of $\mu$ in Eqs. (3.7) and (3.8) we have used the result of the integration of the simulated pair distribution function, i.e.

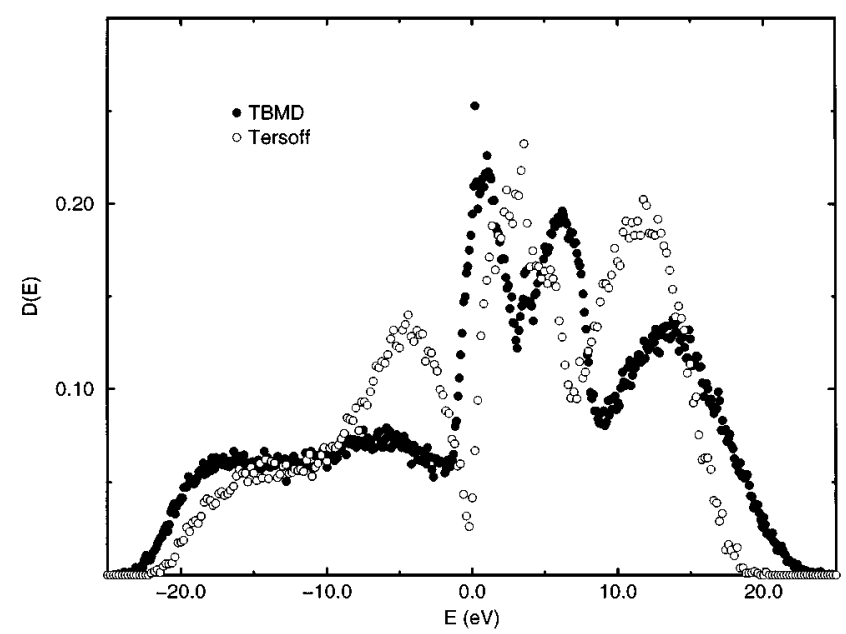

FIG. 4. Total Density of States for liquid Carbon from TBMD (filled circles) and by direct diagonalization over configurations generated by Monte Carlo simulation using Tersoff's potential (hollow circles). 


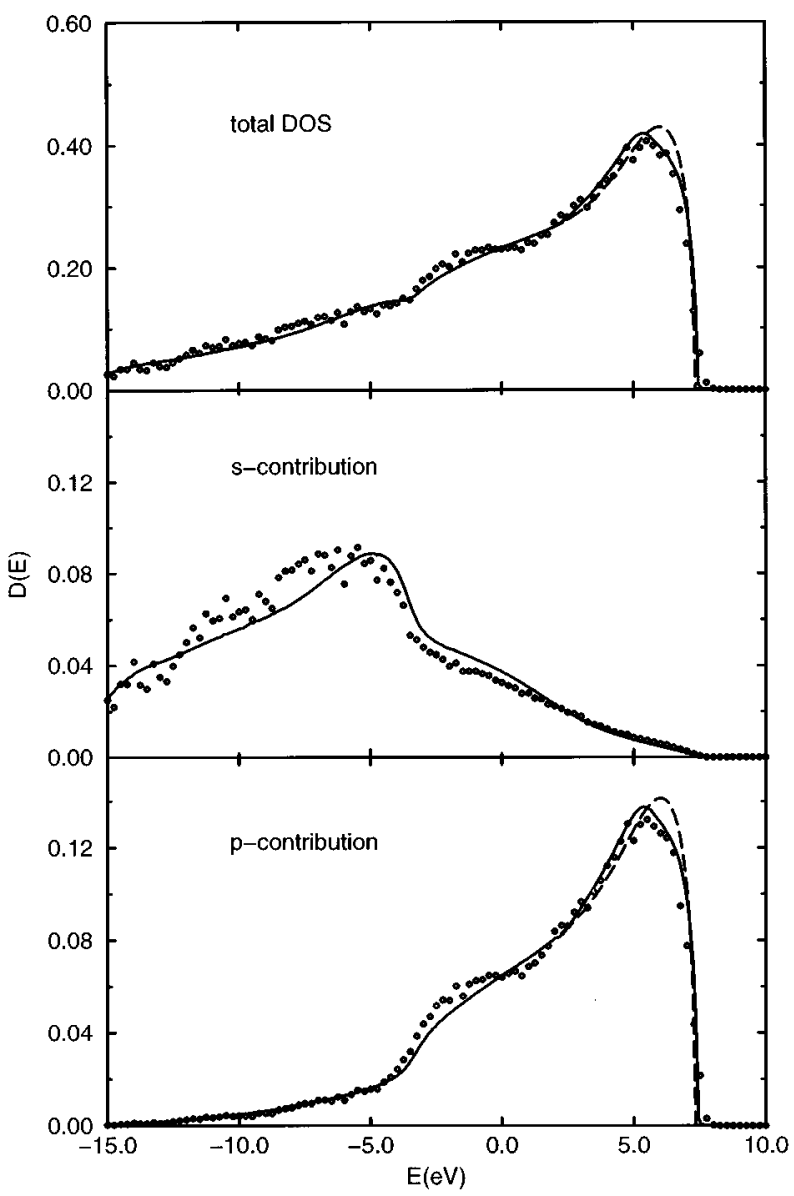

FIG. 5. Total and partial Density of States for liquid Silicon from TBMD (hollow circles), SSCA/EMA (dashed lines) and non-linear theory (solid lines).

$$
\mu=\rho\left(1+4 \pi \rho \int_{0}^{\infty} r^{2}(g(r)-1) d r\right) .
$$

In the case of Silicon (but not for Carbon) this value is extremely low $(\mu / \rho=0.08)$ and as a consequence the effect of the non-linear corrections on the DoS is negligible. However, it is possible to find a value of $\mu$ that introduces corrections in the desired direction. In this case we have found that $\mu=0.5 r_{0}^{-3}$ ( $r_{0}$ is our scaling distance) leads to a perfect agreement with simulation (in the low density limit $\mu=\rho$, and in our case $\rho=0.728 r_{0}^{-3}$ ). In Fig. 6 we have plotted the $\sigma$ and $\pi$ contributions to the $p$ band, and one sees that it is precisely the displacement in the position of the $\sigma$ band that actually brings the whole theoretical prediction to a better agreement with the simulation. In the results presented in Figs. 5 and 6 we have used the TBMD $g(r)$ as input for the theoretical calculations. To illustrate the effect of using the StW pair distribution functions we can see in Fig. 7 a comparison between the SSCA/EMA results obtained using both sets of structural data. We see that the discrepancies are of the same sort as found in Fig. 3.

The situation for Carbon is essentially different. Now $\mu / \rho=0.7$ and the non-linear corrections will be more important. The smaller cutoff radius $(2.1 \AA$ vs. $3.6 \AA$ in Silicon $)$

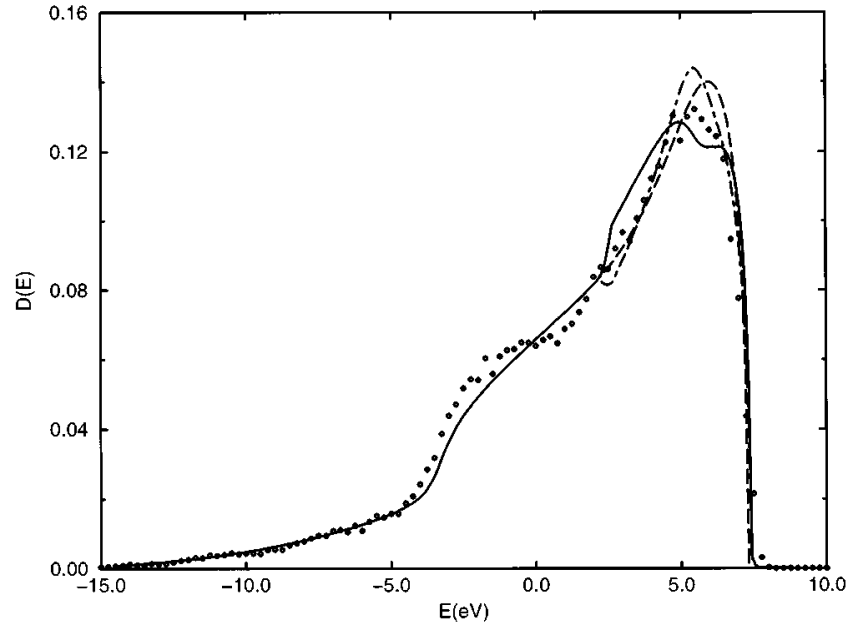

FIG. 6. Total $p p$-contribution to the Density of States in liquid Silicon from TBMD (hollow circles), $p p_{\sigma}$ (solid lines), $p p_{\pi}$ contributions (dash-dotted line) from the non-linear theory and total $p p$ contribution from the SSCA (dashed line). Note that the total $p p$-contribution is normalized to one, so that we have $D^{p p}=\left(D^{p p_{\sigma}}+2 D^{p p} \pi\right) / 3$.

and the much larger values of the hopping parameters introduce strong deviations from the mean field behavior that seems to be characteristic of liquid Silicon. Also, in Carbon the difference between the $p p_{\pi}$ and $p p_{\sigma}$ hopping terms (7 $\mathrm{eV}$ at nearest neighbor separation) is larger than in Silicon $(\approx 4 \mathrm{eV})$. This will inforce a more directional structure with lower coordinations. As mentioned before for this thermodynamic state chain formation will be very much favored. This particular feature we expect is reflected in a large difference between the $p p_{\pi}$ and $p p_{\sigma}$ contribution to the DoS that is not captured by linear approaches.

With these previous considerations in mind, we can now take a look at Fig. 8, where we have plotted the total DoS and the $s$ and $p$ contributions as obtained from the TBMD and the SSCA/EMA with non-linear corrections added (solid

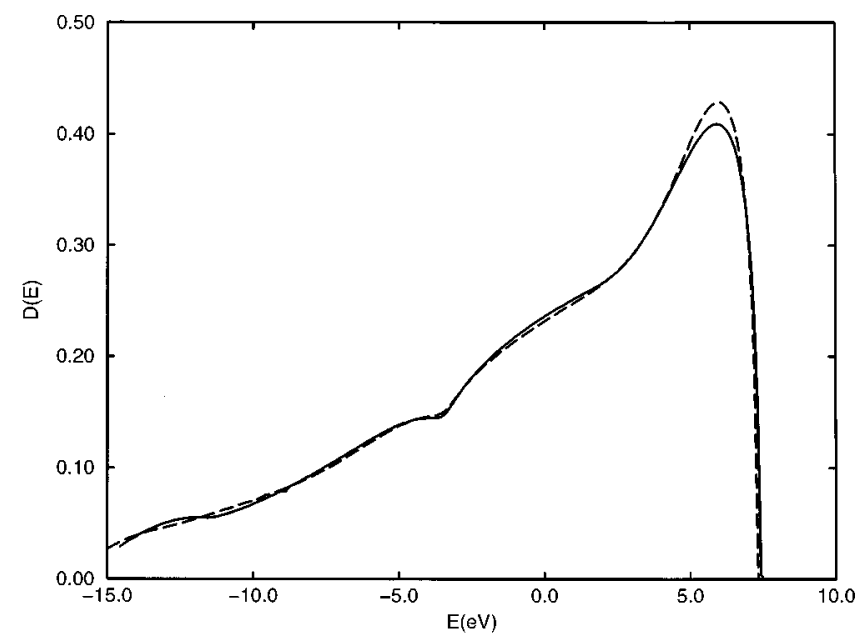

FIG. 7. SSCA/EMA Total Density of States for liquid Silicon with structural input from TBMD (solid line), and from Monte Carlo simulation with StW potential (dashed line). 


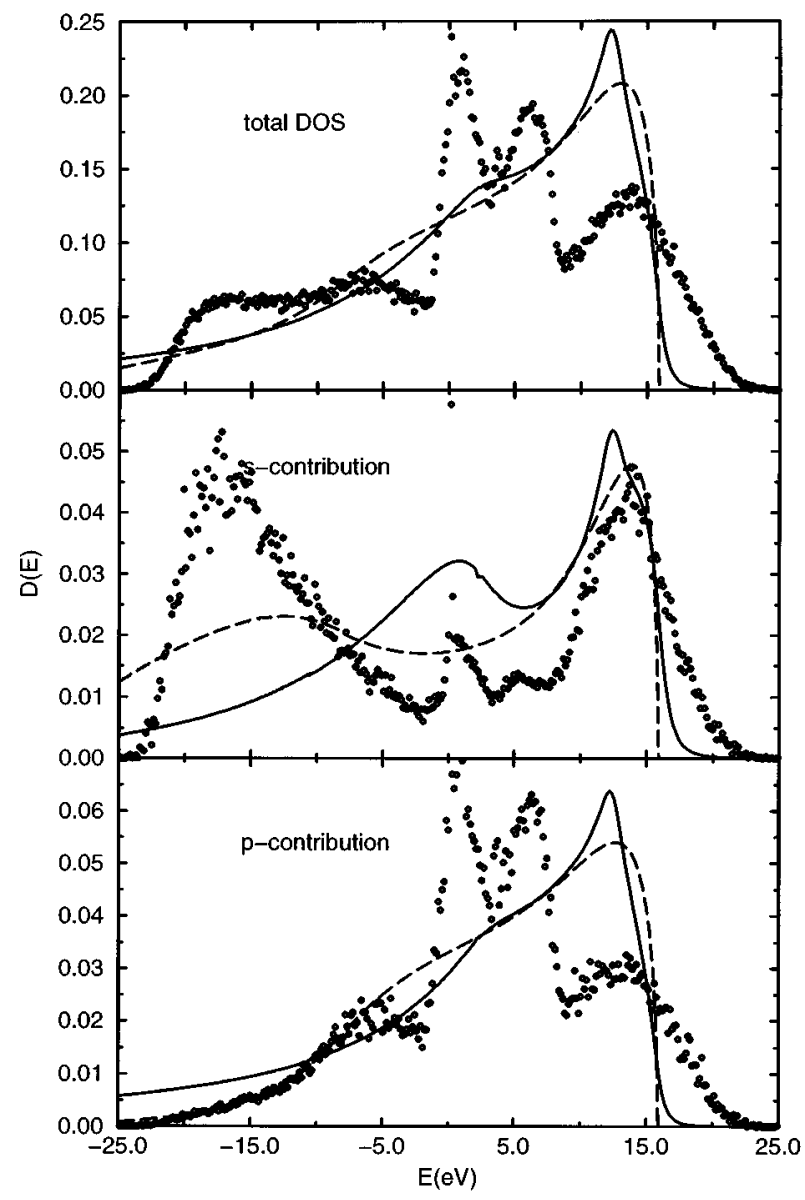

FIG. 8. Total and partial Density of States for liquid Carbon from TBMD (hollow circles), SSCA/EMA (dashed lines) and non-linear theory (solid lines).

line) and without (dashed line). When compared with the good performance of the theory for Silicon, the results now look rather disappointing. The approximation fails to reproduce the considerable symmetry of the band with respect to its center of gravity (close to the on-site energy of the $p$ orbitals, $\varepsilon_{p}=3.71 \mathrm{eV}$ ). Taking into account that the interaction is quasi-nearest neighbors, the symmetry of the band (which is connected with a vanishingly small third moment) can also be ascribed to the formation of coordinations which do not allow for odd number of hops between particles, as is the case in chains, trigonal and tetrahedral coordinations. ${ }^{18}$ The long range of the interactions in silicon bypasses these limitations and leads to a relatively large and negative third moment of the energy distribution. As mentioned above, one may speculate that in order to capture these features properly, three-body correlations will have to be incorporated. For instance, a rescaling of distances in the configurations generated using Tersoff's potential would bring its corresponding pair distribution function to a good agreement with its TBMD counterpart. This would certainly cure the difference of band width observed in Fig. 4 but we have checked that it would not give rise to the splitting of the $p$ peak. This we regard as a clear indication that this feature will only be

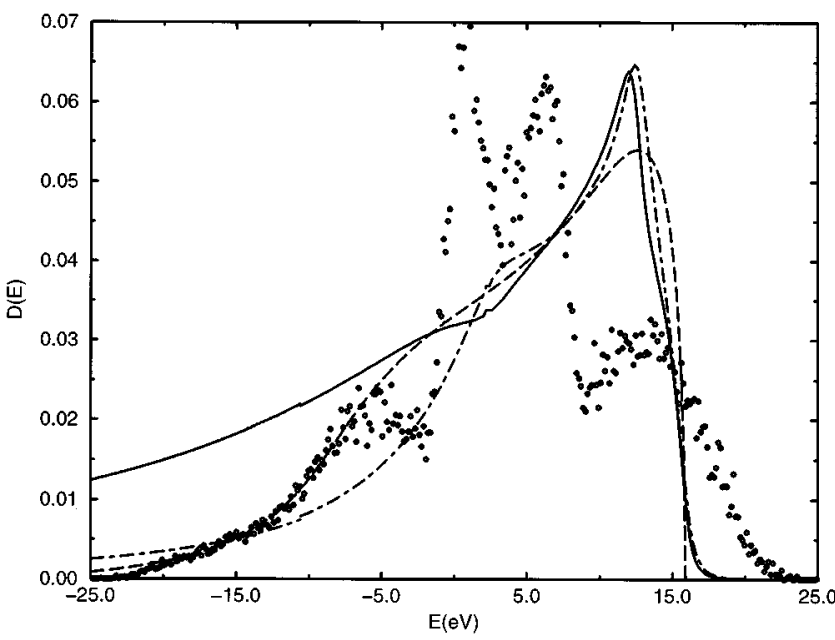

FIG. 9. Total $p p$-contribution to the Density of States in liquid Carbon from TBMD (hollow circles), $p p_{\sigma}$ (solid lines), $p p_{\pi}$ contributions (dash-dotted line) from the non-linear theory and total $p p$ contribution from the SSCA (dashed line). Note that the total $p p$-contribution is normalized to one, so that we have $D^{p p}=\left(D^{p p_{\sigma}}+2 D^{p p \pi}\right) / 3$.

accounted for if the three particle correlation function is incorporated in some way.

Nonetheless, it can be seen that the non-linear corrections account to a certain extent for the wings of the spectrum (in particular the high energy wing of the $s$-band contribution, cf. center of Fig. 8) and the peaks of the $p$-band show some traces of the electronic structure exhibited by the simulation, namely a peak around $13 \mathrm{eV}$ and a shoulder at 5 $\mathrm{eV}$. If we now take a look at the $p p_{\pi}$ and $p p_{\sigma}$ contributions depicted in Fig. 9 we first notice that the non-linear theory predicts large differences between both terms, in contrast with the results for Silicon (Fig. 6). The $\pi$ band presents a shoulder in the vicinity of the central peaks of the simulated DoS at $5 \mathrm{eV}$. Also, the theoretical $\pi$ peak lies on top of the maximum of the TBMD at $13 \mathrm{eV}$, but this is clearly

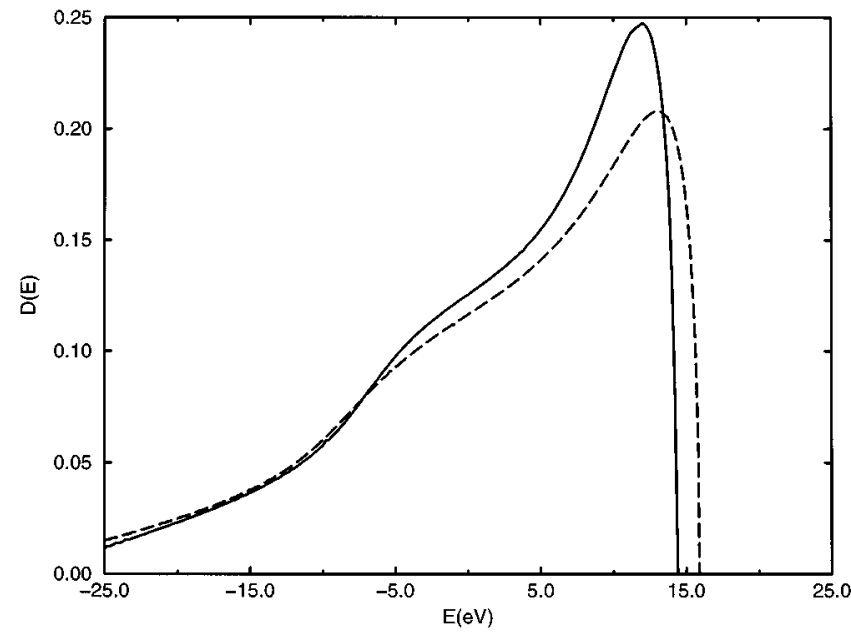

FIG. 10. SSCA/EMA Total Density of States for liquid Carbon with structural input from TBMD (solid line), and from Monte Carlo simulation with Tersoff's potential (dashed line). 
overestimated. At low energy even the linear theory performs somewhat better. As to the use of pair correlations generated using Tersoff's potential we in Fig. 10 compare the SSCA/EMA solution using both TBMD and Tersoff's Monte Carlo $g(r)$. Although the width and height of the bands reflect somehow the different spatial structure, the simulated bands shown in Fig. 4 are more dissimilar, probably due to the effect of the three body correlations that do not enter the theoretical approaches presented herein.

In summary, we have presented results for non-linear corrections to the SSCA/EMA for silicon and carbon. Whereas the corrections lead to accurate results for liquid $\mathrm{Si}$, in the case of Carbon only some qualitative features of the DoS are reproduced. It seems clear that in this case the strongly directional effects of the structure affect the DoS to an extent that can only be accounted for by involving threebody correlation functions.

\section{ACKNOWLEDGMENTS}

The authors wish to acknowledge support from the Austrian-Spanish Program of Acciones integradas under Grant HU1995-0015. We would like to thank Fernando Bresme for providing the code to perform the cluster analysis. This work has been supported in part by the Spanish Dirección General de Investigación Científica y Técnica (DGICYT) under Grant PB94-0112.
${ }^{1}$ E. Lomba, J.L. López-Martín, and G. Kahl, J. Chem. Phys. 105, 7735 (1996).

${ }^{2}$ J.L. López-Martín, E. Lomba, G. Kahl, M.D. Winn, and M. Rassinger, J. Phys. Condensed Matter 9, 3321 (1997).

${ }^{3}$ L. Goodwin, A.J. Skinner, and D.G. Pettifor, Europhys. Lett. 9, 701 (1989).

${ }^{4}$ M.D. Winn and G. Kahl, J. Chem. Phys. 101, 10850 (1994).

${ }^{5}$ Z. Chen and R.M. Stratt, J. Chem. Phys. 97, 5687 (1992).

${ }^{6}$ J.S. Høye and E. Lomba, J. Chem. Phys. 101, 4083 (1994).

${ }^{7}$ J.S. Høye, E. Lomba and J.L. López Martín, J. Chem. Phys. 101, 9042 (1994)

${ }^{8}$ J.S. Høye, J.L. López Martín, and E. Lomba, J. Chem. Phys. 103, 2178 (1995).

${ }^{9}$ D.E. Logan and M.D. Winn, J. Phys. C 21, 5773 (1988).

${ }^{10}$ M. D. Winn and D.E. Logan, J. Chem. Phys. 96, 4818 (1992).

${ }^{11}$ C.Z. Wang, K.M. Ho, and C.T. Chan, Phys. Rev. B 47, 14835 (1993).

${ }^{12}$ F. Stillinger and T.A. Weber, Phys. Rev. B. 31, 5262 (1985).

${ }^{13}$ J. Tersoff, Phys. Rev. Lett. 61, 2879 (1988).

${ }^{14}$ J.S. Høye and G. Stell, J. Chem. Phys. 77, 3731 (1982); J.S. Høye and E. Lomba, ibid. 95, 4502 (1991).

${ }^{15}$ See for instance the TB parameters for Se found in Table I, in J. Robertson, Adv. Phys. 32, 361 (1983).

${ }^{16}$ R. Virkkunen, K. Laasonen, and R.M. Nieminen, J. Phys. Condensed Matter 3, 7455 (1991)

${ }^{17}$ C.H. Xu, C.Z. Wang, C.T. Chan, and K.M. Ho, J. Phys. Condensed Matter 4, 6047 (1992).

${ }^{18}$ For a simple and clear exposition on the qualitative determination of band shapes in terms of lattice coordination and interaction ranges see the discussion in Chap. 4 of A.P. Sutton, Electronic Structure of Materials (Clarendon, Oxford, 1993).

${ }^{19}$ M.D. Winn and G. Kahl, J. Chem. Phys. 100, 7567 (1994). 